This item was submitted to Loughborough's Research Repository by the author.

Items in Figshare are protected by copyright, with all rights reserved, unless otherwise indicated.

\title{
Locust recognition and detection via aggregate channel features
}

PLEASE CITE THE PUBLISHED VERSION

https://doi.org/10.31256/ukras19.30

PUBLISHER

EPSRC UK-Robotics and Autonomous Systems (UK-RAS) Network

VERSION

AM (Accepted Manuscript)

\section{PUBLISHER STATEMENT}

This work is made available according to the conditions of the Creative Commons Attribution-NonCommercialNoDerivatives 4.0 International (CC BY-NC-ND 4.0) licence. Full details of this licence are available at: https://creativecommons.org/licenses/by-nc-nd/4.0/

\section{LICENCE}

CC BY-NC-ND 4.0

\section{REPOSITORY RECORD}

Yi, Dewei, Jinya Su, and Wen-Hua Chen. 2019. "Locust Recognition and Detection via Aggregate Channel Features". figshare. https://hdl.handle.net/2134/37631. 


\title{
Locust Recognition and Detection via Aggregate Channel Features
}

\author{
Dewei Yi, Jinya Su, and Wen-Hua Chen
}

\begin{abstract}
Locust plagues are very harmful for food security, quality and quantity of agricultural products. With this consideration, precise locust detection is significant for preventing locust plagues. To achieve this task, aggregate channel feature (ACF) object detector with parameters optimization is applied to detect locusts. Experiment results show that ACF object detector with optimized parameters can achieve 0.39 for average precision and $\mathbf{0 . 8 6}$ for log-average miss rate. Moreover, ACF is a non-deep method using a simple model to detect objects. That is, the proposed method is promising to be embedded in a real-time locust detection system.
\end{abstract}

\section{INTRODUCTION}

Pests have brought significant negative effects on food security, agricultural economy and quality of agricultural products [1]. Nowadays, pests are usually detected by human beings. Manual monitoring is a labor-intensive job and expensive for large farms. To tackle this problem, computer vision techniques have provided a promising solution for detecting pests.

In [2], edge features (histograms of oriented gradients) are combined with support vector machine which can efficiently identify aphids. Moreover, support vector machines incorporating with selecting suitable region and color index can achieve less than $2.5 \%$ in identifying thrips [3]. Different from conventional image processing techniques, deep learning methods become very popular for pest identification. In [4], a convolutional neural network is trained to detect moths which shows a very promising result. In [5], pest identification is achieved by using deep residual network and its accuracy is $98.67 \%$ for classifying 10 classes. The deep residual learning is also used to classifying pests from complex background [6].

However, most research of pest identification is treated as an image classification problem, which gives a class label for an image and cannot point out the locations and the number of pests in an image. Ideally, pest identification should be treated as an object detection problem. Both locations and the number of pests can be provided. In addition, different regions have planted different cash crops. This causes that main pest species are different in different regions. For example, cotton is the main cash crop in Xinjiang, China. Thus, the main pests in Xinjiang are aphids [7]. Wheat is the main cash crops in Inner Mongolia, China [8]. Thus, the main pests in Inner Mongolia. In this study, we focus on detecting locusts so as helping local farmers to prevent locust plagues. However, the proposed method could be borrowed to detect other pests (such as aphids, moths). Locust detection system should be embedded system and expected to run in real time. Therefore, deep neural network related methods are not very suitable for this application due to their model too complicated. It does not only

This work was supported by the U.K. Science and Technology Facilities Council under Grant ST/N006852/1, ST/N006712/1, and ST/N006836/1. The authors are all with the Department of Aeronautical and Automotive spend a plenty of time for training model but also very slow in predicting new samples.

To this end, the aggregate channel features (ACF) object detection method is used to identify locusts which is a nondeep object detector and both of training and prediction are very fast as shown in [9]. Firstly, three types of feature representations are extracted including color features, gradient magnitude features, and edge features. Secondly, a fast feature pyramids are used to generate regions of interest. Thirdly, AdaBoost classifier [10] is utilized to identify each generated region whether there is a locust inside. Overall, the first step is to extract efficient features. The combination of second step and third step can locate the position of locust in an image.

The rest of the paper is organized as follows: Section II discusses experiment setup and image data acquisition. Then, Section III elaborates the whole framework of ACF locust detector. Next, Section IV provides the experimental results and evaluates the performance. Finally, Section V concludes the paper along with future work.

\section{MATERIALS}

The two key materials related to this research are experiment setup for the locust plague risk estimate and image acquisition. In the experiment setup, the settings of each experimental zone are explained in detail such as light condition, background, and the number of locusts. In image acquisition, it does not only provide the type and parameters of the used digit but also mention the height and direction of photographing images.

\section{A. Experiment setup}

In the experiment, the data of four different risk levels of locust plague are collected in the outdoor environment, which are non-risk, low-risk, middle-risk and high-risk. In addition, the data is collected in different weathers (sunny day and cloudy day) to guarantee the diversity and generalization. Due to limited cover range of camera, the test field is divided into 9 zones. For Zone No. 1, there are 24 locusts inside which is identified as the high-risk of locust plagues. For Zone No. 2, $3,6,8$ and 9, there is no locusts inside which are recognized as the non-risk of locust plagues. For Zone No. 4, there are 6 locusts put inside which is termed as low-risk of locust plagues. For Zone No. 5 and 7, there are 18 locusts inside which are named as the medium-risk of locust plagues. To stay natural scenes, there are grass, dead leaves and locusts in the test field and all the locusts are in living status which can move

Engineering, Loughborough University, Loughborough LE11 3TU, U.K. Email: D.Yi@lboro.ac.uk (Dewei Yi); J.Su2@lboro.ac.uk (Jinya Su); W.Chen@lboro.ac.uk (Wen-Hua Chen). 
randomly. Figure 1 provides the instances of non-risk level, low-risk level, middle-risk level, and high-risk level.

\section{B. Image acquisition}

Images are obtained from an outdoor test field in the Loughborough University, Leicestershire, UK. The image dataset is collected by the Leica dual camera (2MP, CMOS) mounted at the right above each cell of the test field. Thanks to the outdoor environment, the images are all collected under natural light condition. This is due to the color features are varied in different weather conditions. Images from different weather conditions should be used to train a model. Images were captured at $60 \mathrm{~cm}$ distances right above the test field.

\section{ACF DETECTION FRAMEWORK}

The framework of ACF detector contains three key components including feature extraction, feature pyramids, and classifier. Given an image, several feature channels are extracted and then the pixels of each block are added up with pre-smoothing and post-smoothing. Next, an AdaBoost classifier is trained to distinguish object from the background.

\section{A. Feature Extraction}

Before extracting feature channels, an image is presmoothing by a [ $\left.\begin{array}{lll}1 & 2 & 1\end{array}\right] / 4$ filter. This operation can suppress noise and improve the capability of capturing features. In this work, several channels are computed and extracted. These channels are normalized gradient magnitude, histogram of oriented gradients (six channels), and LUV color channels (three channels). After obtaining 10 channels, these channels are divided into $4 \times 4$ blocks and then pixels in each block are summed. Finally, these channels are post-smoothing with a [ $\left.\begin{array}{lll}1 & 2 & 1\end{array}\right] / 4$ filter. The post-smoothing can help aggregate integral scale.

\section{B. Fast Feature Pyramids}

Feature pyramids are used to propose region of interests (ROI). They are multi-scale representations of an image, where channels are computed at every scale. Therefore, computing feature pyramids is a high time-consuming task. To reduce the cost of computation, a fast feature pyramids method is applied to generate ROI, which runs 8 scales at each octave as in [9].

\section{Classifier}

AdaBoost classifier is used to recognize objects. AdaBoost is an ensemble learning algorithm which combines a number of weak classifiers to construct a powerful classifier. With considering the time-efficiency, depth-two trees are used as weak learners in this work. The pseudocode of AdaBoost classifier is presented in Algorithm 1.

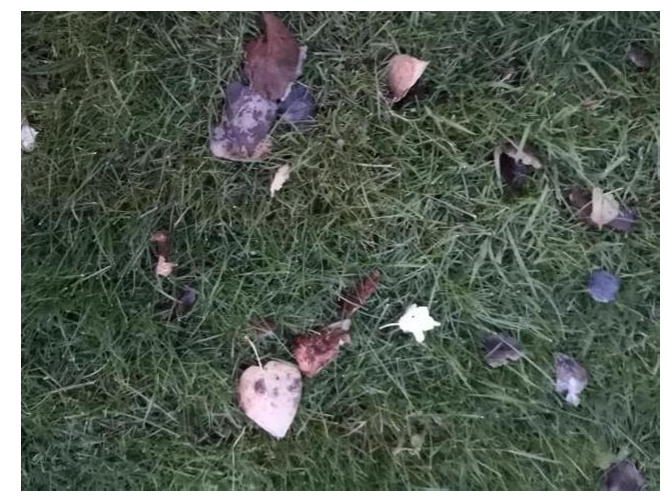

Non-Risk Zone

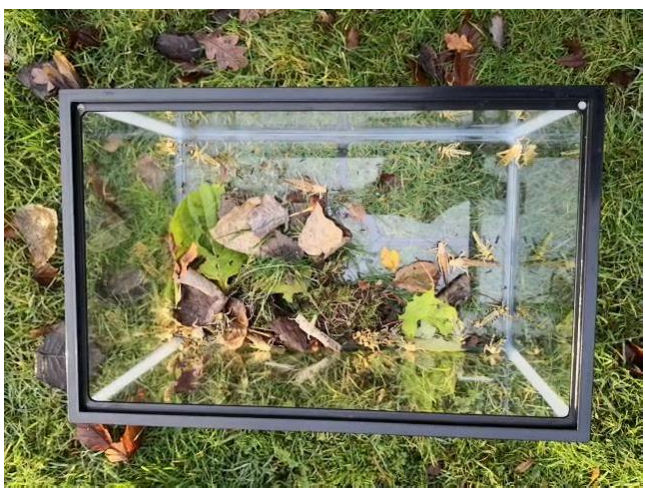

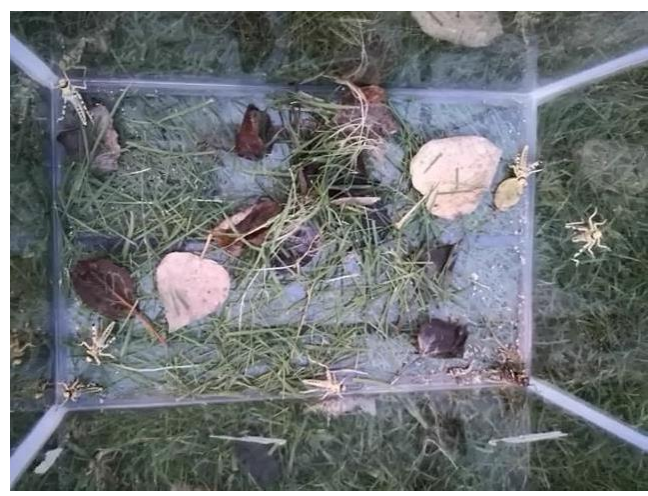

Low-Risk Zone

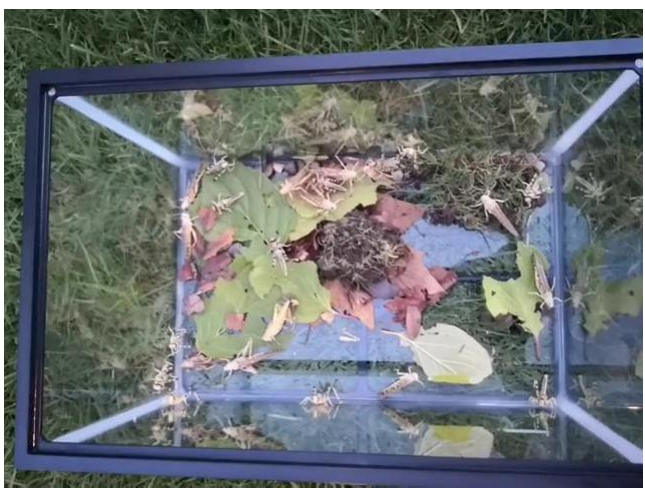

Figure 1. Instances of different locust plague risk level 


\begin{tabular}{l}
\hline \hline Algorithm 1: AdaBoost classifier \\
\hline Initial weights as the same for all depth-two trees \\
For each depth-two tree do \\
Train the current depth-two tree with the given weight. \\
Test the current depth-two tree on the all training data. \\
Reset the current tree weight based on weighted error. \\
Reset weights based on ensemble predictions. \\
End for \\
\hline \hline
\end{tabular}

\section{RESUlTS AND DISCUSSION}

In the section, main results of this work are presented. Before discussing the performance, locust detection dataset and evaluation metrics are introduced.

\section{A. Locust Detection Dataset}

There are 857 images collected by an RGB camera with the resolution of $640 \times 480$. Each image is manually labeled. For enhancing generalization and robustness, images are shuffle before splitting into training dataset and testing dataset. In the experiment, there are $60 \%$ randomly selected as training dataset and the remaining $40 \%$ are selected as testing dataset.

\section{B. Evaluation Metrics}

To evaluate the performance of ACF object detector, several metrics are introduced. They can be mainly divided into correct detection-based metrics and miss detection-based metrics. Correct detection-based metrics includes precision, recall, and average precision. Precision $(\mathrm{P})$ is the number of true positives $(T P)$ over the number of positives. The number of positives is the sum of true positives and false positives (FP).

$$
P=\frac{T P}{T P+F P}
$$

Recall $(R)$ is the number of true positives over the number of true positives plus the number of false negatives $(F N)$.

$$
R=\frac{T P}{T P+F N}
$$

Average precision (AP) summarizes the weighted increase in precision with each change in recall for the thresholds in the precision-recall curve, which is given by

$$
A P=\sum_{i=1}^{n}\left(R_{i}-R_{i-1}\right) P_{i}
$$

where $P_{i}$ and $R_{i}$ are the precision and recall at the $i$-th threshold and $n$ is the total number of thresholds. Therefore, $A P$ is a single number for indicating the object performance with varying thresholds.

Miss detection-based metrics includes miss rate $(M R)$, false positives per image (FPPI), and log-average miss rate. Miss rate is the number of false negatives over the number of true positives plus false negatives.

$$
M R=\frac{F N}{T P+F N}
$$

Log-average miss rate is computed by averaging miss rate at nine FPPI rates evenly spaced in log-space in the range
$10^{-2}$ to $10^{0}$ [11]. Log-average miss rate provides a single value for summaries the miss detection which is convenient for presenting performance straightforwardly.

\section{Performance Evaluation}

The number of stages and negative samples factor are two key parameters needed to be tuned in ACF object detector. A heuristic method is utilized to find the optimal parameters showing in Table I-IV. Because locust detection can be trained offline and then running trained model in real time, the values AP, log-average miss rate and prediction speed is more important compared to training time. The number of trees in AdaBoost classifier is another parameter to be pre-defined. Because the parameter has been tuned in [9], we follow its setting of the parameter which is 2048 . When the overlapping between ground truths and prediction region more than $50 \%$, it is recognized as a true positive. From these Tables, we can see the optimal value of negative samples factor is around 4 and the optimal value of the number of stages is around 6 . We also find that increasing negative samples factor after 4 may obtain more side-effects than benefits. More precisely, ACF object detector with these parameters achieves the best performance regarding to average precision and log-average miss rate.

TABLE I. OPTIMAL ACF PARAMETERS BASED ON AP

\begin{tabular}{|c|c|c|c|}
\hline \multirow{2}{*}{$\begin{array}{c}\text { Number of } \\
\text { Stages }\end{array}$} & \multicolumn{3}{|c|}{ Negative Samples Factor } \\
\cline { 2 - 4 } & 2 & 4 & 6 \\
\hline 2 & 0.08 & 0.08 & 0.11 \\
\hline 4 & 0.32 & 0.35 & 0.31 \\
\hline 6 & 0.30 & $\mathbf{0 . 3 9}$ & 0.34 \\
\hline
\end{tabular}

TABLE II. OPTIMAL ACF PARAMETERS BASED ON LOG-AVERAGE MISS RATE

\begin{tabular}{|c|c|c|c|}
\hline \multirow{2}{*}{$\begin{array}{c}\text { Number of } \\
\text { Stages }\end{array}$} & \multicolumn{3}{|c|}{ Negative Samples Factor } \\
\cline { 2 - 4 } & 2 & 4 & 6 \\
\hline 2 & 0.99 & 0.99 & 0.98 \\
\hline 4 & 0.91 & 0.89 & 0.89 \\
\hline 6 & 0.91 & $\mathbf{0 . 8 6}$ & 0.88 \\
\hline
\end{tabular}

TABLE III. OPTIMAL ACF PARAMETERS BASED ON PREDICTION SPEED (FRAMES PER SECOND)

\begin{tabular}{|c|c|c|c|}
\hline \multirow{2}{*}{$\begin{array}{c}\text { Number of } \\
\text { Stages }\end{array}$} & \multicolumn{3}{|c|}{ Negative Samples Factor } \\
\cline { 2 - 4 } & 2 & 4 & 6 \\
\hline 2 & 2.13 & 2.35 & 2.23 \\
\hline 4 & 2.11 & 2.37 & 2.08 \\
\hline 6 & 2.23 & $\mathbf{1 . 9 6}$ & 0.79 \\
\hline
\end{tabular}

TABLE IV. OPTIMAL ACF PARAMETERS BASED ON TRAINING TIME (SECONDS)

\begin{tabular}{|c|c|c|c|}
\hline \multirow{2}{*}{$\begin{array}{c}\text { Number of } \\
\text { Stages }\end{array}$} & \multicolumn{3}{|c|}{ Negative Samples Factor } \\
\cline { 2 - 4 } & 2 & 4 & 6 \\
\hline 2 & 437 & 670 & 720 \\
\hline 4 & 1147 & 1092 & 1321 \\
\hline 6 & 1834 & $\mathbf{1 7 0 8}$ & 1729 \\
\hline
\end{tabular}

After obtaining appropriate parameters of ACF object detector, we use average precision, log-average miss rate to 
evaluate the performance. With using the selected values of parameters, we can achieve 0.39 of average precision, 0.86 of log-average miss rate. The corresponding Precision-Recall curve and log-average miss rate curve are presented in Figure 2 and 3. Prediction speed and training time based on these parameters setting are 1.96 frames per second and 1708 seconds respectively. Moreover, Figure 4 presents an example of locust detection by ACF object detector.

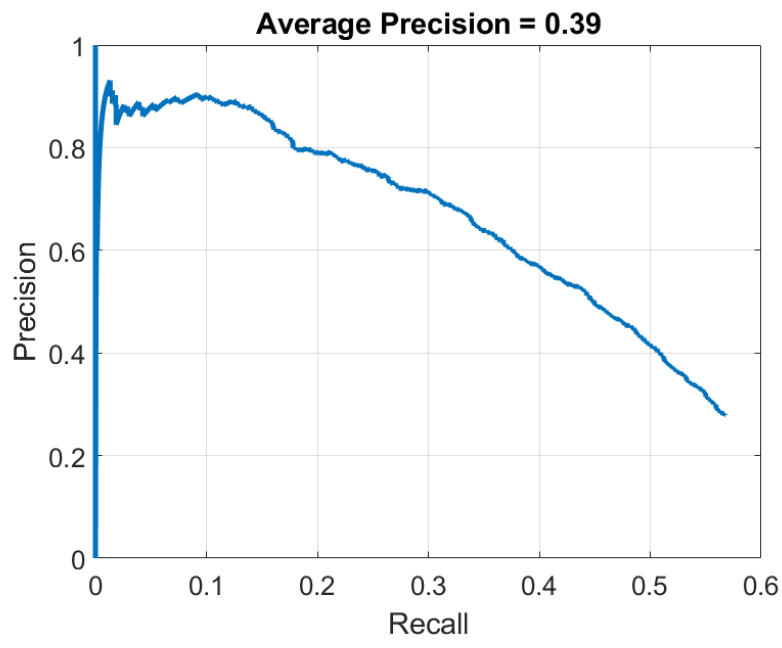

Figure 2. Precision-Recall curve of ACF object detector

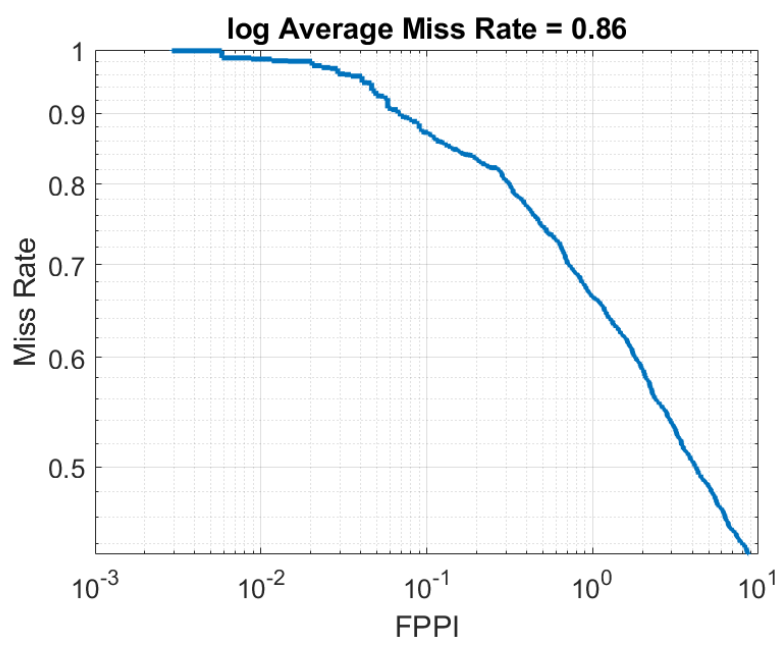

Figure 3. MR-FPPI curve of ACF object detector

\section{CONCLUSION AND FUTURE WORK}

This paper focuses on locust detection problem for preventing locust plagues. This is achieved by using an ACF object detector. Different from previous work on locust identification which only gives a prediction result for a whole image, the ACF object detector can both locate the position and recognize the locusts in an image. The experimental results indicate that $\mathrm{ACF}$ object detector with optimized parameters can achieve 0.39 of average precision and 0.86 of log-average miss rate. Moreover, the prediction speed of ACF object detector is around 2 frames per second by using a single CPU which is a promising method to be embedded in a real- time locust detection system. Tuning the parameters of ACF object detector is a painful job. The parameters are optimized by a heuristic way. In the future, the more advanced parameter optimization methods (such as Bayesian optimization) will be combined into the framework. Moreover, other classifiers will be tested and $k$-fold cross validation will be used. In addition, fast modern deep learning methods (e.g. MobileNets) may be utilized to enhance the performance of object detector.

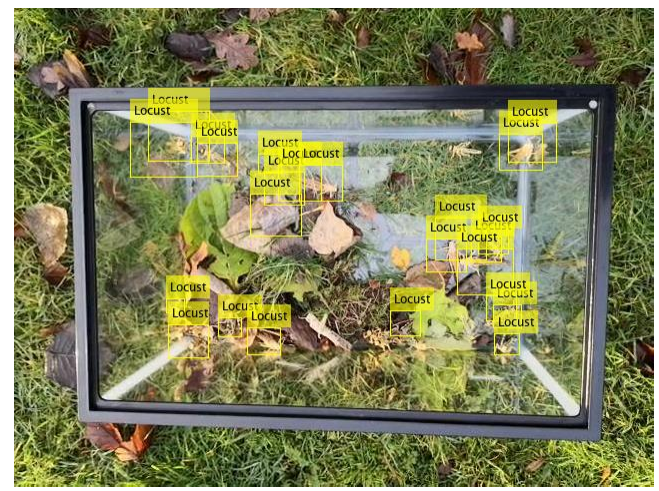

Figure 4. Example of locust detection

\section{REFERENCES}

L. Deng, Y. Wang, Z. Han, and R. Yu, "Research on insect pest image detection and recognition based on bio-inspired methods," Biosyst. Eng., vol. 169, no. 2000, pp. 139-148, 2018.

T. Liu, W. Chen, W. Wu, C. Sun, W. Guo, and X. Zhu, "Detection of aphids in wheat fields using a computer vision technique," Biosyst. Eng., vol. 141, pp. 82-93, 2016.

M. A. Ebrahimi, M. H. Khoshtaghaza, S. Minaei, and B. Jamshidi, "Vision-based pest detection based on SVM classification method," Comput. Electron. Agric., vol. 137, pp. 52-58, 2017. W. Ding and G. Taylor, "Automatic moth detection from trap images for pest management," Comput. Electron. Agric., vol. 123, pp. 17-28, 2016.

C. Wen, D. Wu, H. Hu, and W. Pan, "Pose estimation-dependent identification method for field moth images using deep learning architecture," Biosyst. Eng., vol. 136, pp. 117-128, 2015. X. Cheng, Y. Zhang, Y. Chen, Y. Wu, and Y. Yue, "Pest identification via deep residual learning in complex background," Comput. Electron. Agric., vol. 141, pp. 351-356, 2017.

D. Ma, K. Gorman, G. Devine, W. Luo, and I. Denholm, "The biotype and insecticide-resistance status of whiteflies, Bemisia tabaci (Hemiptera: Aleyrodidae), invading cropping systems in Xinjiang Uygur Autonomous Region, northwestern China," Crop Prot., vol. 26, no. 4, pp. 612-617, 2007.

W. Dong, X. Zhang, X. Zhang, H. Wu, M. Zhang, E. Ma, and J. Zhang, "Susceptibility and potential biochemical mechanism of Oedaleus asiaticus to beta-cypermethrin and deltamethrin in the Inner Mongolia, China," Pestic. Biochem. Physiol., vol. 132, pp. 47-52, 2016.

[9] P. Dollar, R. Appel, S. Belongie, and P. Perona, "Fast feature pyramids for object detection," IEEE Trans. Pattern Anal. Mach. Intell., vol. 36, no. 8, pp. 1532-1545, 2014.

[10] Q. Yao, D. xiang Xian, Q. jie Liu, B. jun Yang, G. qiang Diao, and J. Tang, "Automated counting of rice planthoppers in paddy fields based on image processing," J. Integr. Agric., vol. 13, no. 8, pp. 1736-1745, 2014.

[11] P. Dollár, C. Wojek, B. Schiele, and P. Perona, "Pedestrian detection: An evaluation of the state of the art," IEEE Trans. Pattern Anal. Mach. Intell., vol. 34, no. 4, pp. 743-761, 2012. 